\title{
Fast Intra Bunch Train Charge Feedback for FELs based on Photo Injector Laser Pulse Modulation
}

\author{
Tomasz Kozak, Bernd Steffen, Sven Pfeiffer and Siegfried Schreiber
}

\begin{abstract}
Bunch charge variations in Free Electron Lasers such as the Free Electron Laser in Hamburg (FLASH) or the European X-Ray Free Electron Laser (E-XFEL) impacts the longitudinal phase space distribution of the electrons resulting in different bunch peak currents, pulse duration and pulse shapes. The electron bunches are generated by short ultraviolet laser pulses impinging onto a photocathode inside a radio frequency (RF) accelerating cavity. At FLASH, bursts of bunches up to 800 pulses with an intra train repetition rate of $1 \mathrm{MHz}$ are used and even higher repetition rates for the E-XFEL (up to $4.5 \mathrm{Mhz}$ ) are planned. Charge variations along these bunch-trains can be caused by variations of the laser pulse energies, instabilities of the accelerating fields in the RF cavity and time dependent effects in the photoemission process. To improve the intra bunch-train charge flatness and to compensate train-to-train fluctuations a dedicated digital control system, based on the Micro Telecommunication Computing Architecture (MicroTCA.4) standard, was designed, implemented and successfully tested at the FLASH. The system consists of a bunch charge detection module which analyzes data from toroid system and provides the input signal for the controller which drives a fast UV-Pockels Cell installed in the optical path of the photo-cathode laser. The Pockels cell alters the laser polarization and thus the transmission through a polarizer. The modulation of UV laser pulse energy with an iterative learning feed-forward minimizing the repetitive errors from bunch-train to bunch-train and a fast feedback algorithm implemented in a Field Programmable Gate Array (FPGA) allows for fast tuning of bunch charge inside the bunch-train. In this paper a detailed description of the system and first measurement results are presented.
\end{abstract}

Index Terms-DESY, European XFEL, control systems, Photo-Injector Laser System, MTCA, FPGA

\section{INTRODUCTION}

M ODERN Free Electron Lasers (FELs) as the Free Electron Laser in Hamburg (FLASH)[1] or the European X-Ray Free Electron Laser (E-XFEL)[2] are driven by a high energy electron beam generated by a superconducting accelerator operated in pulsed mode. A complex photo-injector system is often exploited as a source of high density electron bunches for the accelerator. In case of FLASH, the Photo-Injector consists of the drive laser (Photo-Injector Laser, PIL) and the RF-gun[3]. Short laser pulses of intensive ultra-violet (UV) light illuminate photocathode placed at the backplane of the first half cell of the normal conducting 1.5 cell RF gun. Electron bunches produced by photo emission process are

Work supported by DESY MSK and MFL groups.

T. Kozak, B. Steffen, S. Pfeiffer and Siegfried Schreiber are with the Deutsches Elektronen-Synchrotron, Hamburg, Germany. e-mail: \{tomasz.kozak, bernd.steffen, sven.pfeiffer, siegfried.schreiber\}@desy.de. accelerated by intense electric field $(\sim 50 \mathrm{MV} / \mathrm{m})$ in order to mitigate space charge effects degrading the bunch emittance. UV laser pulses at around $260 \mathrm{~nm}$ with energy of about $30 \mu \mathrm{J}$ per pulse are required for $\mathrm{Cs}_{2} \mathrm{Te}$ photocathode used in the system to produce electron bunches with a charge up to $3 \mathrm{nC}$. The FELs operated in pulsed mode need an electron source able to generate hundreds of electron bunches to take advantage from high acceleration efficiency. The FLASH photo-injector operated in standard mode at $10 \mathrm{~Hz}$ repetition rate is able to generate a train of up to 800 bunches at an intratrain repetition rate of maximum $1 \mathrm{MHz}$. In enhanced mode, intra-train repetition rate can be increased from $1 \mathrm{MHz}$ to $3 \mathrm{MHz}$ providing up to 2400 bunches in $800 \mu \mathrm{s}$ RF flattop, but in this case burst rate is limited to $5 \mathrm{~Hz}$ due to limited cooling capabilities. Charge stability variations along the bunch train impacts beam properties as longitudinal phase space distribution, bunch peak current or bunch shape. Therefore, high stability and flatness over the train is a needed condition for stable accelerator operation and FEL light generation. There are a few sources of charge instabilities in bunch trains such as:

- variations in laser pulses energies

- $\quad$ instabilities in RF field of the gun

- time dependent effects in photo emission process

Caused by this, noticeable charge oscillations and charge slope creation along a bunch train in the range from $0.5 \%$ to $3 \%$ were observed at FLASH. In order to compensate such fluctuations and to stabilize the bunch charge a dedicated digital control system has been designed and installed for the FLASH photo-injector.

\section{SYSTEM OVERVIEW}

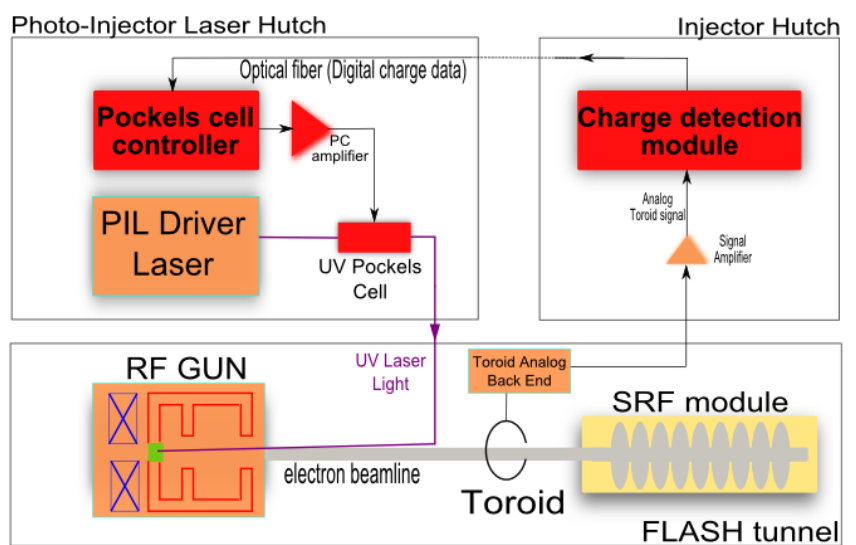

Fig. 1. Intra Bunch Train Charge Feedback System block diagram. Red blocks are components additionally installed for the charge stabilization system. 
The Intra Bunch Train Charge Feedback system principle block diagram is presented on Fig. 1. System is composed of three main subsystems:

- Charge detection module

- Pockels cell controller

- $\quad$ Pockels cell and amplifier

The charge detection module is placed in the Low Level Radio Frequency (LLRF) hutch where the signals from toroid system are available. The analog signal coming from the toroid placed in the accelerator beamline is amplified and shaped by the toroid analog front end and later sampled by fast ADCs. The charge of individual bunches in the bunch train is calculated by a dedicated algorithm implemented in an FPGA. Digitized data are sent using a fast optical link to the injector laser hutch where laser is located. The data is received by controller board and processed by a control algorithm also implemented in an FPGA. The control signal is used to drive the fast UV Pockels cell (BBO Pockels cell by LEYSOP LTD, England) installed in optical path of the laser on the path to the RF gun photocathode.

\section{SYSTEM IMPLEMENTATION}

\section{A. Charge detection}

Charge data within the same bunch train is required to close the fast feedback loop. Therefore, a real-time charge detection algorithm has been implemented in the Virtex 5 FPGA of the commercially available SIS8300 Advanced Mezzanine Card (AMC). Analog signals from the toroid are feed through a SIS8900 Rear Transition Module (RTM) to the AMC board where fast ADCs are placed. The detection algorithm takes as input signal the raw ADC data stream sampled at $81.25 \mathrm{MHz}$ with a clock generated by Local Oscillator synchronized to the $\mathrm{RF}$ field of the accelerator $(1.3 \mathrm{GHz})$. The integrated value which is proportional to the bunch charge is send via a fast low latency fiber link operated with $3.125 \mathrm{~Gb} / \mathrm{s}$ to the PhotoInjector hutch, when a toroid pulse is detected. Data consistency is protected by cyclic redundancy check (CRC) code. Charge detection board is inserted in the same MicroTCA.4 crate as the LLRF test system.

\section{B. Pockels cell controller}

Integrated charged data are received by a controller implemented in Virtex 6 FPGA placed on SIS8300L AMC card. Simplified block diagram of the firmware is presented in Fig. 2.

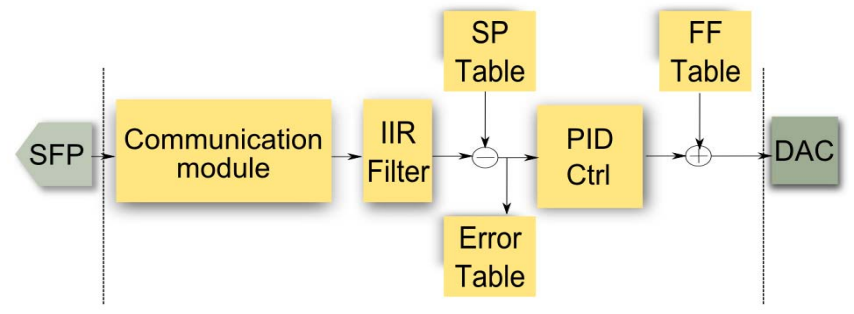

Fig. 2. Simplified block diagram of controller firmware.
The data received from fiber link is validated by Communication Module. CRC checksum is calculated and source of data is checked. Next, the signal is filtered by configurable infinite impulse response (IIR) filter, subtracted from a pre-defined set-point and this error is transmitted to the PID controller input. Control signal generated by PID is used to drive Digital-Analog Converter (DAC) mounted on SIS8300L AMC. DAC output voltage is limited from $-1 \mathrm{~V}$ to $1 \mathrm{~V}$. In order to provide correct Pockels cell operation voltage range, the SIS8300L DAC front panel connector is attached to the commercial Pockels cell driver (EYSOP LTD, England) with an amplification factor of 125 .

\section{High level software}

To monitor and control the system two DOOCS servers has been developed - Charge Detection Server, mainly for monitoring and setup of charge detection SIS8300 AMC, and Pockels Cell Controller server performing additional algorithms. The most important one is Learning Feedforward table calculation.

\section{RESULTS}

First system tests have been performed in March 2016. The system was installed in beam path of photo injector laser 2 used to drive the FLASH 1 accelerator. System influence on the 300 bunches within the train is presented in Fig. 3.

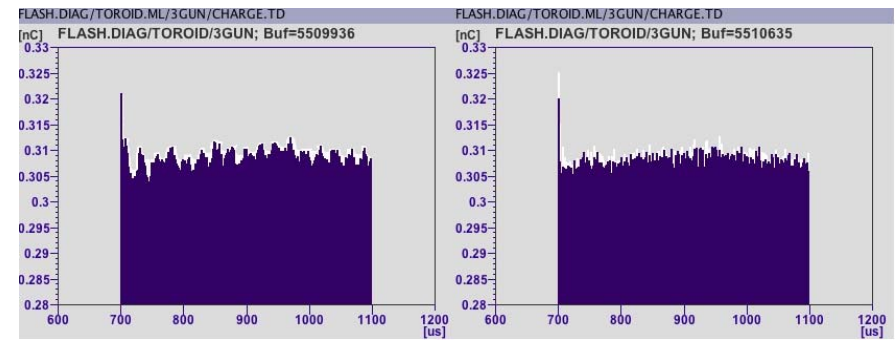

Fig. 3. Charge fluctuation registered in FLASH 1 accelerator with Intra Bunch Train Feedback System off (left picture) and on (right picture). Only fast feedback loop enabled.

One can easily observe that the charge fluctuations are reduced using a fast intra-train feedback loop. More detailed measurements show that the intra-train instabilities are reduced from $1.23 \%$ to $0.95 \%$ giving an improvement of $23 \%$ currently limited by the noise level of the charge detection. More detailed system description and obtained results are planned to be published soon.

\section{REFERENCES}

[1] W. Ackermann et al., "Operation of a free-electron laser from the extreme ultraviolet to the water window", Nature Photonics 1, 336 (2007).

[2] M. Altarelli, "The European X-ray free-electron laser facility in Hamburg", Nuclear instruments \& methods in physics research / B 269, 2845-2849 (2011)

[3] I. Will, H. I. Templin, S. Schreiber, and W. Sandner, "Photoinjector drive laser of the FLASH FEL", Optics Express 19, 23770 (2011).

[4] M. Werner, T. Lensch, J. Lund-Nielsen, R. Neumann, D. Noelle, and N. Wentowski, „A Toroid baser Bunch Charge Monitor System with Machine Protection Features for FLASH and XFEL", Proceedings of IBIC2014, Monterey, CA, USA, WEPF02 